\title{
Obesity-related differences between women and men in brain structure and goal-directed behavior
}

\section{Annette Horstmann 1,2*, Franziska P. Busse ${ }^{3}$, David Mathar',2, Karsten Müller', Jöran Lepsien', Haiko Schlögl, Stefan Kabisch ${ }^{3}$, Jürgen Kratzsch ${ }^{4}$, Jane Neumann ${ }^{1,2}$, Michael Stumvoll ${ }^{2,3}$, Arno Villringer ${ }^{1,2,5,6}$ and Burkhard Pleger ${ }^{1,2,5,6}$}

\author{
Department of Neurology, Max Planck Institute for Human Cognitive and Brain Sciences, Leipzig, Germany \\ 2 Integrated Research and Treatment Center Adiposity Diseases, Leipzig University Medical Center, Leipzig, Germany \\ ${ }^{3}$ Department of Medicine, University Hospital Leipzig, Leipzig, Germany \\ ${ }_{4}^{4}$ Institute for Laboratory Medicine, Clinical Chemistry and Molecular Diagnostics, University Hospital Leipzig, Leipzig, Germany \\ ${ }^{5}$ Clinic for Cognitive Neurology, University Hospital Leipzig, Leipzig, Germany \\ ${ }^{6}$ Mind and Brain Institute, Berlin School of Mind and Brain, Humboldt-University, Berlin, Germany
}

\section{Edited by:}

Hauke R. Heekeren, Max Planck Institute for Human Development, Germany

\section{Reviewed by:}

Alain Dagher, Montreal Neurological Institute and Hospital, Canada Kirk I. Erickson, University of Illinois, USA

\section{${ }^{*}$ Correspondence:}

Annette Horstmann, Department of Neurology, Max Planck-Institute for Human Cognitive and Brain Sciences, Stephanstrasse 1A, D-04103 Leipzig, Germany.

e-mail:horstmann@cbs.mpg.de
Gender differences in the regulation of body-weight are well documented. Here, we assessed obesity-related influences of gender on brain structure as well as performance in the lowa Gambling Task. This task requires evaluation of both immediate rewards and long-term outcomes and thus mirrors the trade-off between immediate reward from eating and the long-term effect of overeating on body-weight. In women, but not in men, we show that the preference for salient immediate rewards in the face of negative long-term consequences is higher in obese than in lean subjects. In addition, we report structural differences in the left dorsal striatum (i.e., putamen) and right dorsolateral prefrontal cortex for women only. Functionally, both regions are known to play complimentary roles in habitual and goal-directed control of behavior in motivational contexts. For women as well as men, gray matter volume correlates positively with measures of obesity in regions coding the value and saliency of food (i.e., nucleus accumbens, orbitofrontal cortex) as well as in the hypothalamus (i.e., the brain's central homeostatic center). These differences between lean and obese subjects in hedonic and homeostatic control systems may reflect a bias in eating behavior toward energy-intake exceeding the actual homeostatic demand. Although we cannot infer from our results the etiology of the observed structural differences, our results resemble neural and behavioral differences well known from other forms of addiction, however, with marked differences between women and men. These findings are important for designing gender-appropriate treatments of obesity and possibly its recognition as a form of addiction.

Keywords: gender difference, voxel-based morphometry, obesity, brain structure, lowa gambling task, reward system

\section{INTRODUCTION}

The regulation of body-weight and energy-intake is a complex process involving humoral as well as central homeostatic and hedonic systems. Gender-based differences in the regulation of body-weight covering these domains are reported in the literature. The prevalence of obesity is slightly higher in women (in Germany, where this study was conducted, women $20.2 \%$, men $=17.1 \%$, World Health Organization, 2010) and differences between genders regarding the biological regulation of body-weight have been described for gastrointestinal hormones (Carroll et al., 2007; Beasley et al., 2009; Edelsbrunner et al., 2009) and for eating-related social and environmental factors, as well as for dietary behavior (Rolls et al., 1991; Provencher et al., 2003).

A recent study showed that obesity risk-factors for women and men differ profoundly despite having the same effect on bodyweight: for men, most of the difference between groups with high and low health risk was explained by variability in eating competence (a score covering eating attitudes, food acceptance, internal regulation, and contextual skills such as meal planning) and the conscious restriction of food intake. For women, the inability to resist emotional cues and uncontrolled eating explained most of the group-differences (Greene et al., 2011).
These observations hint at fundamental differences in the way women and men process food-related information and control food intake, which is supported by evidence of partly separated neural mechanisms in response to food and in the control of eating behavior for both genders (Parigi et al., 2002; Smeets et al., 2006; Uher et al., 2006; Wang et al., 2009). However, since both men and women can become obese, neither of these ways seems to protect from excess weight gain.

In this study we investigated two aspects of gender-related differences in obesity. First, using voxel-based morphometry (VBM), we assessed differences in brain structure in lean and obese men and women. Second, we explored possible gender-related differences in cognitive control over eating behavior using a modified version of the Iowa Gambling Task (Bechara et al., 1994).

A recent study using functional MRI found gender-related differences in ad libitum energy-intake following 6 days of eucaloric feeding as well as in food-related brain activation for normal weight subjects (Cornier et al., 2010). In this study, activation in dorsolateral prefrontal cortex (DLPFC) correlated negatively with energyintake, but with increased activation levels in women as compared to men. The authors suggested that these greater prefrontal neural 
responses in women reflect increased cognitive processing related to executive function, such as guidance or evaluation of eating behavior. In obesity, however, impairment of these control mechanisms may contribute to excess energy-intake.

To investigate possible gender-related differences in cognitive control over eating behavior in obesity, we used a modified version of the IGT. This task requires evaluation of both immediate rewards and long-term outcomes and thus mirrors the trade-off between immediate reward from eating and the long-term influence of overeating on body-weight. Assuming that obese subjects prefer high immediate rewards even in the face of long-term negative outcome, we focused our investigations on card deck B. In this deck high immediate rewards are accompanied by infrequent but high punishments leading to a negative long-term outcome. In order to contrast each of the other decks with deck B individually, we presented only two instead of four alternative card decks at any time. Hypothesizing that obesity differentially affects cognitive control over behavior in men and women, we expected to find effects of both gender and obesity on behavioral measures in the IGT.

Voxel-based morphometry is a valuable tool in identifying differences in the brain's gray matter (GM) structure related not only to diseases but also to task performance (Sluming et al., 2002; Horstmann et al., 2010). Moreover, GM density and structural parameters of white matter have recently been shown to change rapidly in response to altered behavior such as mastering a new skill - in other words, showing that the brain is a plastic organ (Draganski et al., 2004; Scholz et al., 2009; Taubert et al., 2010). Therefore, adaptations in functional circuits due to altered behavior such as persistent overeating could be reflected in the brain's GM structure.

First pioneering studies investigating the structure of the brain in obesity showed obesity-related differences in various brain systems (Pannacciulli et al., 2006, 2007; Taki et al., 2008; Raji et al., 2010; Schäfer et al., 2010; Walther et al., 2010; Stanek et al., 2011) Although being very insightful in identifying brain structures which are different in obesity, those studies did not investigate possible gender-related effects. One study reported an influence of both gender and obesity on the diffusion properties of white matter (Mueller et al., 2011).

We studied the relationship between brain structure and obesity [as measured by body mass index (BMI) as well as leptin] using VBM in both men and women in a normal aged, healthy sample, matched for gender and BMI distribution. Given the above mentioned gender differences in the processing of food-related information, we hypothesized to find gender-dependent in addition to gender-independent correlates of obesity in brain structure.

\section{MATERIALS AND METHODS \\ SUBJECTS}

We included 122 healthy Caucasian subjects. We matched males and females according to distribution and range of BMI as well as age [61 females (premenopausal), BMI (f) $=26.15 \mathrm{~kg} /$ $\mathrm{m}^{2}$ (SD 6.64, 18-44), BMI $(\mathrm{m})=27.24 \mathrm{~kg} / \mathrm{m}^{2}(\mathrm{SD} 6.13,19-43)$, $\chi^{2}=35.66(25), p=0.077$; age $(\mathrm{f})=25.11$ years $(\mathrm{SD} 4.43,19-41)$, age $(\mathrm{m})=25.46$ years $(\mathrm{SD} 4.25,20-41), \chi^{2}=11.02(17), p=0.856$; see Figure 1 for distribution of BMI and age within both groups]. Inclusion criteria were age between 18 and 45. Exclusion criteria were hypertension, dyslipidemia, metabolic syndrome, depres-

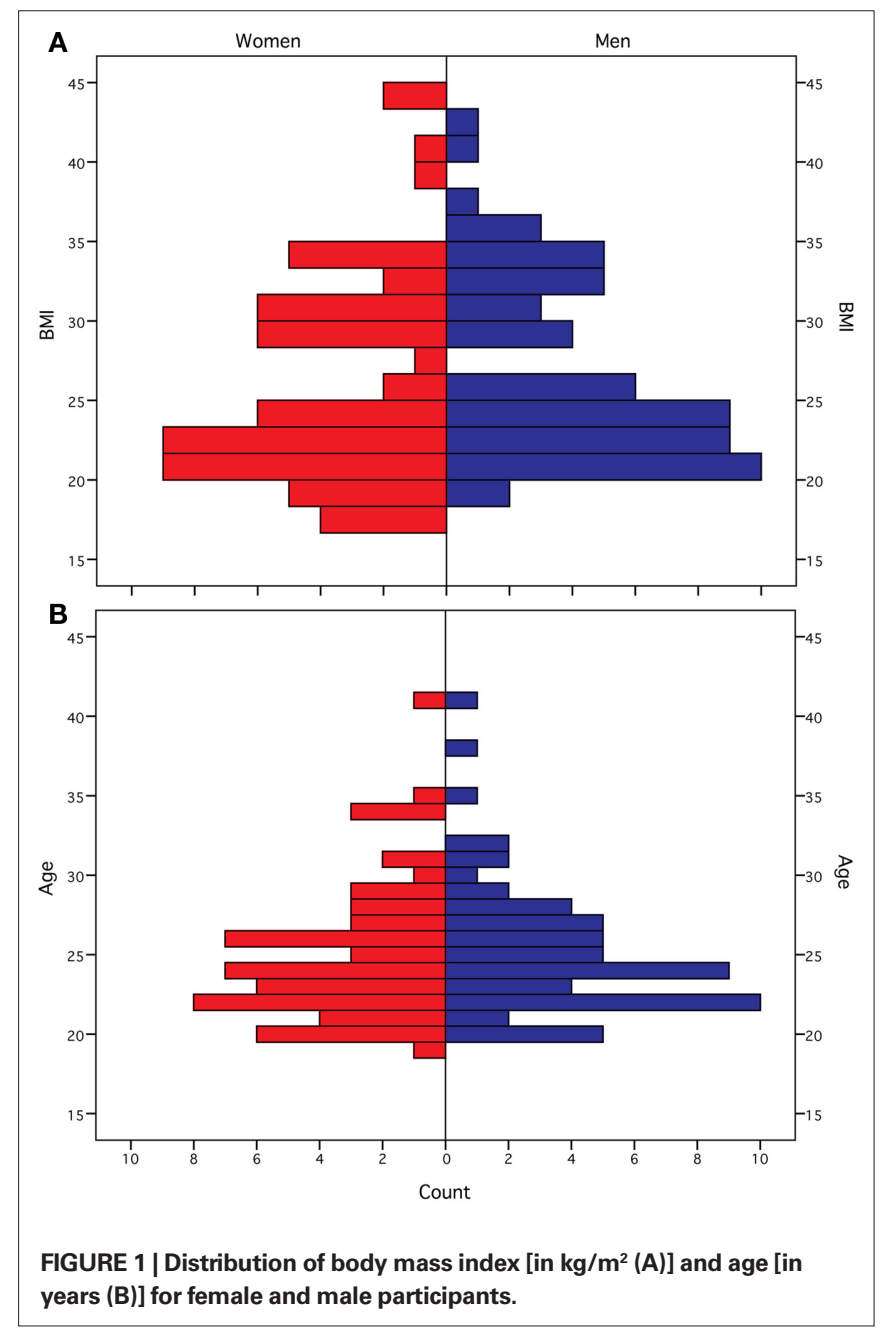

sion (Beck's Depression Inventory, cut-off value 18), a history of neuropsychiatric diseases, smoking, diabetes mellitus, conditions which are contraindications to MR-imaging and abnormalities in the T1-weighted MR scan. The study was carried out in accordance with the Declaration of Helsinki and approved by the local ethics committee of the University of Leipzig. All subjects gave written informed consent before taking part in the study.

\section{MRI ACQUISITION}

T1-weighted images were acquired on a whole-body 3T TIM Trio scanner (Siemens, Erlangen, Germany) with a 12-channel headarray coil using a MPRAGE sequence $[\mathrm{TI}=650 \mathrm{~ms}$; TR = $1300 \mathrm{~ms}$; snapshot FLASH, TRA $=10 \mathrm{~ms}$; TE $=3.93 \mathrm{~ms}$; alpha $=10^{\circ}$; bandwidth $=130 \mathrm{~Hz} /$ pixel (i.e., $67 \mathrm{kHz}$ total); image matrix $=256 \times 240$; FOV $=256 \mathrm{~mm} \times 240 \mathrm{~mm}$; slab thickness $=192 \mathrm{~mm} ; 128$ partitions; $95 \%$ slice resolution; sagittal orientation; spatial resolution $=1 \mathrm{~mm} \times 1 \mathrm{~mm} \times 1.5 \mathrm{~mm} ; 2$ acquisitions $]$.

\section{IMAGE PROCESSING}

SPM5 (Wellcome Trust Centre for Neuroimaging, UCL, London, UK; http://www.fil.ion.ucl.ac.uk/spm) was used for T1-weighted image pre-processing and statistical analysis. MR images were 
processed using the DARTEL approach (Ashburner, 2007) with standard parameters for VBM running under MatLab 7.7 (Mathworks, Sherborn, MA, USA). All analyses were performed on bias-corrected, segmented, registered (rigid-body transformation), interpolated isotropic $(1.5 \mathrm{~mm} \times 1.5 \mathrm{~mm} \times 1.5 \mathrm{~mm})$, and smoothed (FWHM $8 \mathrm{~mm}$ ) images. All images were warped based on the transformation of the group-specific DARTEL template to the GM prior image provided by SPM5 to meet the standard stereotactical space of the Montreal Neurological Institute (MNI). GM segments were modulated (i.e., scaled) by the Jacobian determinants of the deformations introduced by normalization to account for local compression and expansion during transformation.

\section{STATISTICAL ANALYSES}

The following statistical models were evaluated: a full-factorial design with one factor (gender) and two levels (women and men), including BMI as a covariate centered on the factor mean with no interaction. Additional models included interactions between either BMI or central leptin level and gender to examine the differential effects of these covariates within both groups. All statistical models included covariates for age and total gray and white matter volumes to account for the confounding effects of age and brain size. Results were considered significant at a voxel-wise threshold of $p<0.001$ with an additional cluster-level threshold of $p<0.05$ (FWE-corrected, whole brain). Effectively, this combined voxel- and cluster-level statistic reflects the probability that a cluster of a given size, consisting only of voxels with $p<0.001$, would occur by chance in data of the given smoothness. Results were further corrected for non-isotropic smoothness (Hayasaka et al., 2004).

\section{ANALYTICAL PROCEDURES}

Leptin, an adipocyte-derived hormone, is well known to correlate with the percentage of body fat (Considine et al., 1996; Marshall et al., 2000). Central effects for leptin have been extensively described (Fulton et al., 2006; Hommel et al., 2006; Farooqi et al., 2007; Dileone, 2009). We therefore included estimated central leptin level (i.e., the natural logarithm of peripheral leptin, Schwartz et al., 1996) in addition to BMI as measure of obesity. Serum leptin concentration (Enzyme-linked immunosorbent assay, Mediagnost, Reutlingen, Germany) was determined for a subsample $\left[n=56\right.$ (24 females), BMI (f) $=27.29 \mathrm{~kg} / \mathrm{m}^{2}$ (SD 6.67, 19-44), BMI $(\mathrm{m})=30.13($ SD 6.28, 20-43); age $(\mathrm{f})=25.33$ years $($ SD 5.27, 19-41), age $(\mathrm{m})=25.19$ years $(\mathrm{SD} 4.5,20-41)]$.

\section{MODIFIED IOWA GAMBLING TASK Participants}

Sixty-five healthy participants were tested with the modified Iowa Gambling Task [34 females, 15 lean (mean BMI $21.9 \mathrm{~kg} / \mathrm{m}^{2} \pm 2.2$; mean age 24.1 years \pm 2.8 ) and 19 obese (mean BMI $35.4 \mathrm{~kg} /$ $\mathrm{m}^{2} \pm 3.9$; mean age 25.4 years \pm 3.4 ); 31 males, 16 lean (mean BMI $23.8 \mathrm{~kg} / \mathrm{m}^{2} \pm 3.2 ;$ mean age 25.2 years \pm 3.8 ) and 15 obese (mean BMI $33.5 \mathrm{~kg} / \mathrm{m}^{2} \pm 2.4$; mean age 26.7 years \pm 4.0$\left.)\right]$. Subjects with a BMI greater than or equal to $30 \mathrm{~kg} / \mathrm{m}^{2}$ were classified as being obese. The four subgroups were matched according to their educational background. One obese female subject was excluded from the analysis due to a thyroid hypofunction.

\section{Experimental procedure}

The modified IGT version and behavioral data acquisition were implemented in Presentation 14.1 (Neurobehavioral Systems Inc., Albany, CA, USA). Our modified task version was similar in its general deck composition to the original IGT (Bechara et al., 1994). Decks A and B were disadvantageous, leading to a long-term loss and decks $\mathrm{C}$ and $\mathrm{D}$ resulted in a positive long-term outcome. Our modifications of the task only pertained to the number of different card decks presented simultaneously and to the gain/loss frequency and gain/loss size in each deck. Participants had to choose between two alternative card decks in each block (e.g., deck B + C). Deck $\mathrm{A}$ and $\mathrm{C}$ had a gain/loss frequency of 1:1 with an immediate gain of +100 (+70 respectively) and an immediate loss of $-150(-20$ respectively). Decks B and D had a gain/loss frequency of 4:1 and yielded immediate rewards of +100 ( +50 respectively) and losses in the amount of -525 ( -75 respectively). Hence, deck A and B led to an overall net loss while deck $\mathrm{C}$ and $\mathrm{D}$ led to a net gain.

In every trial, two card decks with a question mark in between were shown on the screen, indicating that subjects had to choose one card. The question mark was replaced by a white cross after participants made their choices. In each trial, participants had to make their decision in less than $3 \mathrm{~s}$. If the subjects failed to select a card within this limit, a smiley with a question mark mouth appeared and the next trial started. These trials were discarded.

Participants completed 90 trials subdivided into 3 randomized blocks $(\mathrm{AB} / \mathrm{BC} / \mathrm{BD})$ of 30 trials each. After each block, a break of $30 \mathrm{~s}$ was introduced, in which subjects were informed that the card decks presented would be different in the following block. Analogously to the original IGT, subjects were told to maximize their outcome via advantageous deck choices.

For motivational issues, participants were paid a bonus of up to $6 €$ in addition to the baseline payment according to their performance in the task.

\section{Data analysis}

All results were computed with PASW Statistics 18.0 (IBM Corporation, Somers, NY, USA). The number of cards drawn from deck $B$ was analyzed with respect to obesity and gender differences including age as a covariate in the general linear model. In addition, learning curves were investigated using a repeated-measures ANOVA. Further ANOVAs to obtain separate group effects for both genders with respect to obesity were performed. The correlation between BMI and preference for deck B was computed using a linear model.

\section{RESULTS GRAY MATTER STRUCTURE}

To explore correlates of obesity in brain structure, we used DARTEL forVBM of the whole brain (Ashburner, 2007) based on T1-weighted MRI. Detailed results are shown in Figure 2 and Table 1. We found a positive correlation between BMI and gray matter volume (GMV) in the medial posterior orbitofrontal cortex (OFC), the nucleus accumbens (NAcc) bilaterally, the hypothalamus, and the left putamen (i.e., dorsal striatum, peak voxels $p<0.05$, FWE-corrected for multiple comparisons at voxel-level) when both men and women were included in the analysis (see Figure 2). Performing the same analysis within the equally sized groups $(n=61)$ of women and 




Table 1| Correlations between gray matter and measures of obesity.

\begin{tabular}{|c|c|c|c|c|c|c|c|c|}
\hline GM correlation & Region & Laterality & $x$ & $y$ & $z$ & $z$ value & $p$ & Sign $r$ \\
\hline \multirow[t]{3}{*}{ BMI whole group } & OFC/NAcc & Left & -10 & 18 & -14 & 4.41 & $<0.0001$ & + \\
\hline & OFC/NAcc & Right & 18 & 18 & -14 & 5.55 & $<0.0001$ & + \\
\hline & Hypothalamus & - & 3 & 6 & -15 & 4.09 & $<0.0001$ & + \\
\hline BMI women & Putamen & Left & -30 & -2 & 10 & 4.75 & $<0.0001$ & + \\
\hline \multirow[t]{5}{*}{ Central leptin women } & OFC/NAcc & Left & -16 & 6 & -15 & 3.76 & $<0.0001$ & + \\
\hline & OFC/NAcc & Right & 18 & 10 & -16 & 3.57 & $<0.001$ & + \\
\hline & Putamen & Left & -22 & 18 & 4 & 4.20 & $<0.0001$ & + \\
\hline & Fornix & - & -2 & -4 & 21 & 4.07 & $<0.0001$ & + \\
\hline & DLPFC & Right & 39 & 39 & 32 & 4.04 & $<0.0001$ & - \\
\hline
\end{tabular}

Statistical values are given for cluster peaks, coordinates are in Montreal Neurological Institute (MNI) space. GM, gray matter; sign r gives the direction of correlation; OFC, orbitofrontal cortex; NAcc, nucleus accumbens.

men separately, we obtained comparable results for women but not for men: In particular, we found a significant positive correlation between GMV in OFC/NAcc and BMI in both groups (Figure 3 top row, females $r=0.48, p<0.001$, males $r=0.48, p<0.001$ ) but a significant correlation between the GMV in the putamen and BMI for women only (Figure 3 middle row, women $r=0.51, p<0.001$; men $r=0.003, p=0.979$ ).
Obese subjects are known to show elevated peripheral leptinlevels, a circulating adipocyte-derived hormone that correlates strongly with the amount of body fat (Marshall et al., 2000; Park et al., 2004). Hence, elevated leptin-levels reflect the amount of excess body fat. As an elevated BMI does not necessarily reflect excess body fat, we used leptin as an additional measure of the degree of obesity to make sure that a high BMI in our sample 


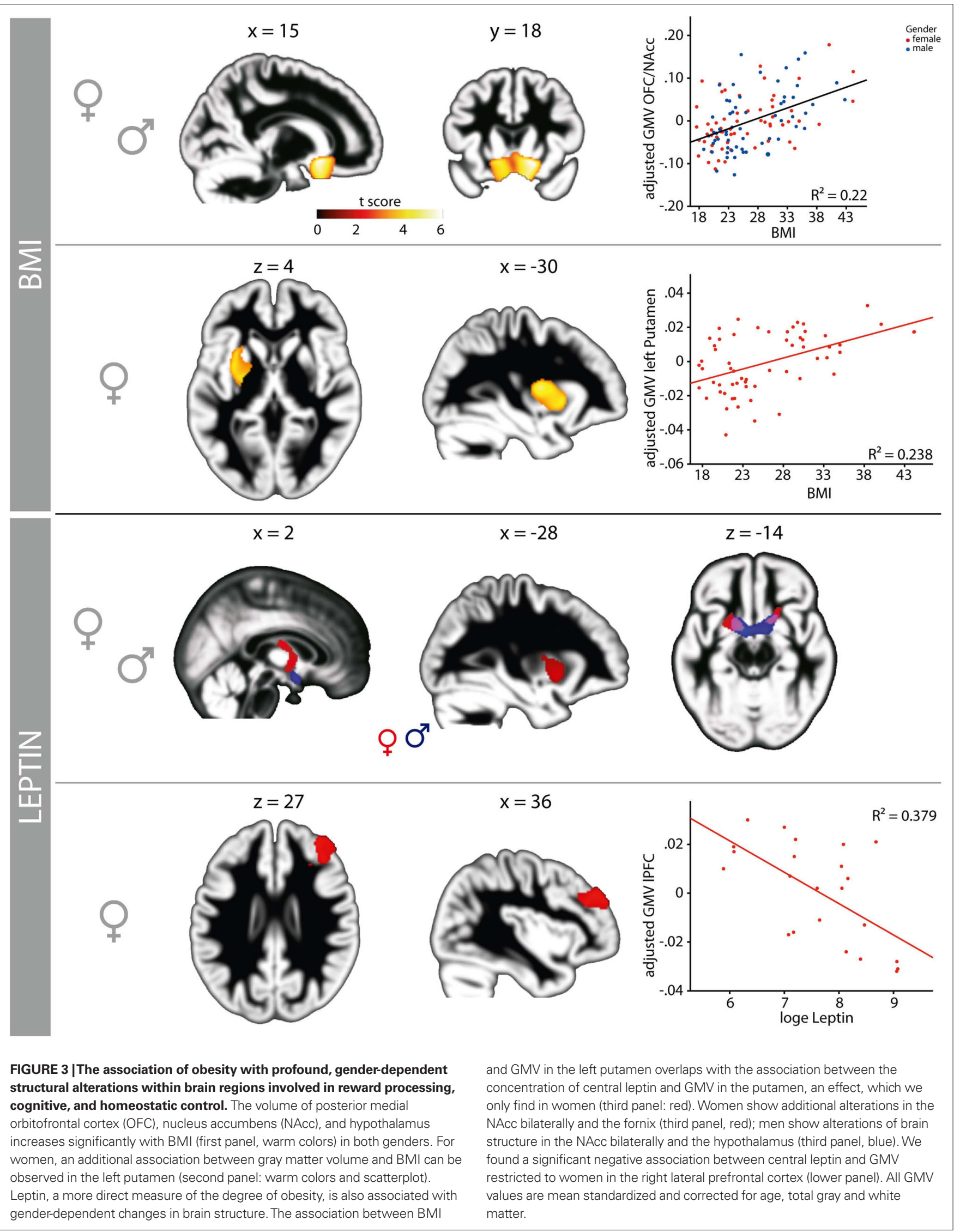


indeed reflects excess body fat rather than excess lean mass. We found that women had a higher absolute serum leptin concentration as compared to men [women $30.92 \mathrm{ng} / \mathrm{ml}$ (SD 26.07), men $9.65 \mathrm{ng} / \mathrm{ml}$ (SD 8.66), $p<0.0001$ ]. An ANCOVA revealed a significant interaction between BMI ( 2 levels: normal weight $\leq 25$; obese $\geq 30)$, gender, and serum leptin concentration $\left(F_{1,41}=16.92\right.$, $p<0.0001)$.

For both men and women, we found a positive correlation between leptin and GMV in the NAcc and ventral striatum bilaterally (women $r=0.56, p=0.008$; men $r=0.51, p=0.005$ ) as well as in the hypothalamus (Figure 3 third row). Only women show additional leptin-related structural differences in the left putamen and the fornix (Figure 3, areas shown in red in third row). The clusters in the NAcc and putamen show a substantial overlap with the regions identified by correlating BMI with GMV (Figure 3 first to third row). Moreover, only for women did we find an inverse (i.e., negative) correlation between leptin-levels and GMV in the right DLPFC ( $r=-0.62, p<0.001$; Figure 3, bottom row).

\section{RELATIONSHIP BETWEEN GAMBLING BEHAVIOR, GENDER, AND OBESITY}

In the IGT, deck B conveys high immediate rewards with each card but low frequency high losses, ultimately resulting in a negative long-term outcome. Hence, the options in deck B mirror the conflict between very salient immediate rewards and the achievement of long-term goals. In the present version of the Iowa Gambling Task, obese women chose significantly more cards from deck B when contrasted with each advantageous deck (i.e., C or D) than lean women across all trials $\left(F_{1,32}=8.68, p=0.006\right)$. We found no difference between lean and obese women when contrasting the two disadvantageous decks (i.e., A and B). Additionally, there was a significant correlation between BMI and the total number of cards chosen from deck B for women (Figure 4A). Comparing lean with obese men we found neither a significant difference for the total number of cards chosen from deck B $\left(F_{1,29}=0.51, p=0.48\right)$, nor a significant correlation with BMI.

In order to test differences in learning behavior between lean and obese participants, we analyzed choices of deck B over time. Over the course of learning, obese women showed no adjustment in choice behavior. In contrast, for lean women we observed a gradual decrease in the preference for cards from deck B (see Figure 4B). Thus, obese women did not adapt their behavior toward an overall advantageous outcome compared to lean women. Analysis of learning behavior only revealed a significant effect for obesity in women $\left(F_{1,30}=6.61, p=0.015\right)$ but not in men.

This effect of gender was particularly pronounced in the last phase of learning (i.e., trials 25-30), where we observed a significant interaction between gender and obesity for choice behavior on deck B $\left(F_{1,59}=6.10 ; p=0.02\right)$. Here, obese women chose more than twice as many cards from deck B as lean women $\left(F_{1,33}=17.97\right.$, $p<0.0001)$. For male subjects, no significant difference was observed (Figure 4C, $\left.F_{1,29}=0.13, p=0.72\right)$. Moreover, a correlation analysis showed a strong correlation $(r=0.57, p<0.0001)$ between BMI and the number of cards chosen from deck B in the last block for women. Again, no significant correlation was observable for men $(r=0.17, p=0.35)$.
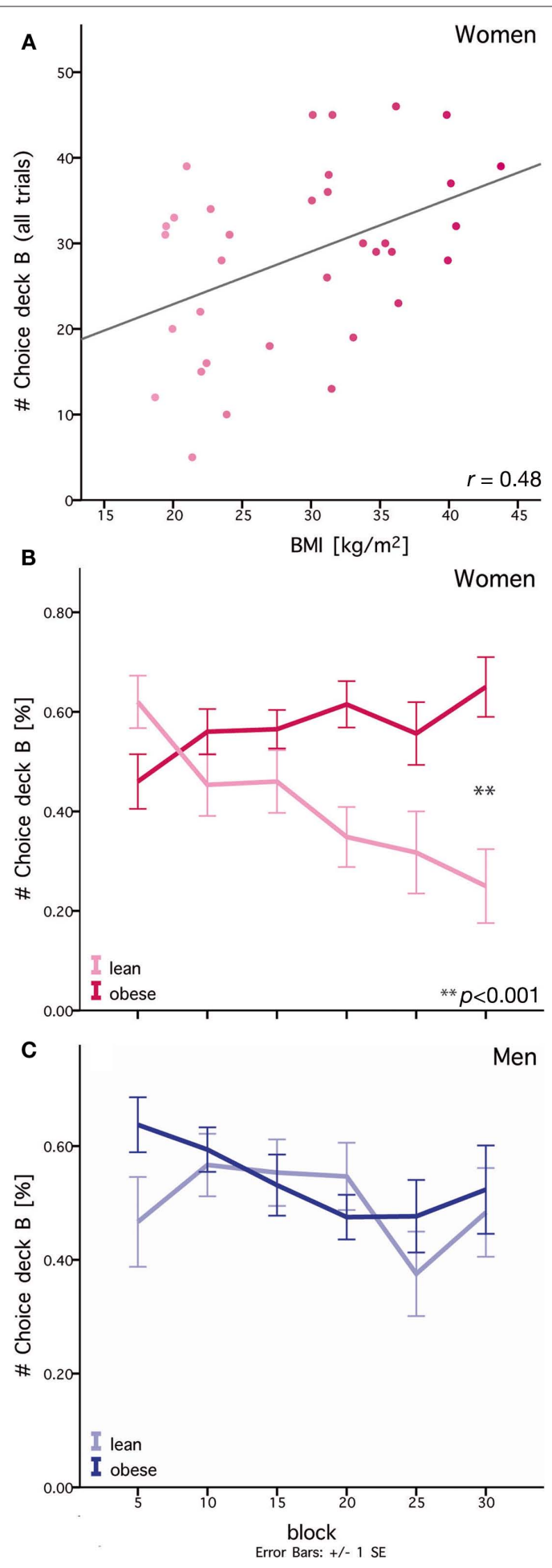

FIGURE 4 | Differences in lean and obese women in their ability to adjust choice behavior to match long-term goals. (A) Preference for deck B over all trials correlates with BMI within the group of women. Gray line: linear regression. (B) Difference between lean and obese women in choice behavior during learning. Lean women learn to gradually select fewer cards from deck B. In contrast, obese women continue to select cards from deck B. Each block consists of five trials. (C) No difference between lean and obese men in choice behavior is observable during learning. Each block consists of five trials. 


\section{DISCUSSION}

For both men and women, we show a correlation between GMV and measures of obesity in the posterior medial OFC (mOFC) and within the ventral striatum (i.e., the NAcc) which is in line with previously reported group-differences in GM when comparing lean to obese subjects (Pannacciulli et al., 2006). The interplay between these two regions is crucial for evaluating motivationally salient stimuli (such as food) and relaying this information for the purpose of decision-making. Functionally, these regions code the saliency and subjective value of stimuli (Plassmann et al., 2010). In bulimia nervosa (BN), a condition where eating behavior but NOT BMI differs from normal, GMV of the same structures is higher in patients than in controls (Schäfer et al., 2010). This suggests that the structure of these regions is either affected by or is a predisposition for altered eating behavior instead of being physiologically determined by the percentage of body fat.

In addition to mOFC and NAcc, both genders showed a correlation between brain structure and obesity within the hypothalamus. The hypothalamus is a key region controlling hunger, satiety, eating behavior as well as energy expenditure and possesses direct connections to the reward system (Philpot et al., 2005). We hypothesize that these differences between lean and obese subjects in both the hedonic and homeostatic control systems may reflect one key feature of obesity, namely a bias in eating behavior toward more hedonic food choices where energy-intake exceeds the actual homeostatic demand.

In women only, we additionally show correlations between GMV and measures of obesity (BMI as well as central leptin-levels) in the dorsal striatum (i.e., left putamen) and in the right DLPFC. Interestingly, these structures play important, complimentary roles in habitual (automatic) and goal-directed (cognitive) control of behavior in motivational contexts: The mOFC and NAcc signal the preference for and the expected value of reward, the putamen in the dorsolateral striatum is thought to code (amongst many other functions) behavioral contingencies to obtain a specific reward, and the DLPFC provides goal-directed cognitive control over behavior (Jimura et al., 2010). Goal-directed behavior is characterized by a strong dependency between the likelihood of the response and the anticipated outcome (e.g., Daw et al., 2005). In contrast, habitual (or automatic) behavior is characterized by a strong link between a stimulus (e.g., food) and a response (e.g., its consumption). In this case, the probability of the response is barely influenced by the outcome of the action itself, whether it may be in the short term (satiation) or long-term (obesity).

Recently, Tricomi et al. (2009) investigated the neural basis of the emergence of habitual behavior in humans. They applied a paradigm well known to elicit habit-like behavior in animals, and showed that basal ganglia activations (notably in the dorsal putamen, see also Yin and Knowlton, 2006) increased across training, suggestive of a role in a progressive reinforcement learning process. The functional role of the putamen in this context may be to establish cue-driven sensory-motor loops, and thus to help automate excessively learned behavior. Furthermore, action-outcome representations in the mOFC also continued to increase in anticipation of reward throughout all sessions. These results show that habitual responding does not result from a decrease in the anticipation of reward outcomes across learning, but from strengthening of stimulus-response links (Daw et al., 2005; Frank and Claus, 2006; Frank, 2009). In the context of obesity, Rothemund et al. (2007) previously demonstrated, using an fMRI-paradigm, that BMI predicts activation in the putamen during viewing of high-caloric food in women. Furthermore, Wang et al. (2007) have shown a gender difference in the putamen regarding changes in CBF in response to stress: Stress in women primarily activated the limbic system, including the ventral striatum and putamen.

The basal ganglia are strongly interconnected with the PFC (Alexander et al., 1986), establishing integrative cortico-striatocortical pathways linking reward-based learning, motivational context and goal-directed behavior (e.g., Draganski et al., 2008). Miller and Cohen (2001) stated that cognitive control over behavior is predominantly provided by the PFC. They conclude that activity in the PFC subserves the selection of a response, which is appropriate in a given situation even in the face of a stronger (e.g., more automatic/ habitual or desirable) alternative. It has recently been demonstrated that the DLPFC guides anticipatory implementation of behavioral goals within working memory in rewarding and motivational contexts (Jimura et al., 2010). Gender-related differences for activity in this region in the context of food and control of eating behavior have also been demonstrated recently by Cornier et al. (2010). They found that right DLPFC activation in response to hedonic food was only apparent in women, while men showed a deactivation. Activation in DLPFC was negatively correlated with subsequent ad libitum energyintake, suggesting a specific role of this cortical region in the cognitive control of eating behavior. If one assumes functional relevance of altered brain structure, the negative relationship between GMV in the right DLPFC and obesity found in the present study may be interpreted as an impairment in the ability to adjust current actions to long-term goals or, in other terms, a loss of cognitive control over eating behavior in obese as compared to lean women.

Applying a simplified version of the Iowa Gambling Task, a learning task with very salient immediate rewards conflicting with the achievement of long-term goals, we observed that lean women decreased their choice of deck B over time, while obese women did not. This finding may support the functional relevance of the observed differences in brain structure in rewarding contexts. Differences on the classical IGT between morbidly obese and healthy-weight subjects have been shown recently (Brogan et al., 2011). However, the results of the aforementioned study were not analyzed for influences of gender. Our findings point to a higher sensitivity to immediate rewards in obese than in lean women, accompanied by a possible lack of inhibitory goal-directed control. Further evidence for an impact of obesity on decision-making has been provided by Weller et al. (2008), who found that obese women showed greater delay-discounting than lean women. Interestingly, they did not find differences in delay-discounting behavior between obese and lean men, which corroborates our gender-specific results. Another study, which only included women, tested the impact of obesity on the effectiveness of response inhibition and found that obese women showed less effective response inhibition than lean women in a stop-signal task (Nederkoorn et al., 2006). In the context of eating behavior, less effective behavioral inhibition in combination with a higher sensitivity to immediate rewards may facilitate overeating, especially when faced with a constant supply of highly palatable food. 
Koob and Volkow (2010) recently suggested key roles of the striatum, the OFC, and the PFC in the preoccupation/anticipation stage and in disrupted inhibitory control in addiction. They observe that the transition to addiction (i.e., compulsory drug taking) involves neuroplasticity in several central structures and conclude that these neuro-adaptations are a key factor to vulnerability for developing and maintaining addictive behavior. Hence, our findings may support the hypothesis that obesity resembles a form of addiction (Volkow and Wise, 2005), but with marked differences between women and men.

Although we cannot infer functional differences from our findings in brain structure, it is conceivable that the structural differences have also functional relevance. This is further supported by experiments showing modulatory effects of centrally acting gut hormones such as ghrelin, PYY, and leptin on these regions (Batterham et al., 2007; Farooqi et al., 2007; Malik et al., 2008). Dynamic changes in brain structure have recently been shown to parallel learning processes as well as to accompany detrimental progressions such as atrophy (Draganski et al., 2004; Horstmann et al., 2010; Taubert et al., 2010). Since our study, although crosssectional, included a set of healthy young subjects, we hope to have minimized the possibly confounding effects such as aging and maximized the obesity-specific effects of interest. To our knowledge, we are the first to describe a positive correlation between GM and markers of obesity. The discrepancy between the results published on brain structure and obesity so far and our findings might be explained by differences in sample composition and study design. Studies reporting negative correlations between obesity and brain structure either involved subjects that were considerably older than the subjects in our sample or included subjects with an overall great age range (Taki et al., 2008; Raji et al., 2010; Walther et al., 2010). Detrimental effects of obesity may emerge later in life, so that our findings may describe the early phase of changes in brain structure related to obesity. Also, as these studies were not designed to investigate gender differences,

\section{REFERENCES}

Alexander, G. E., DeLong, M. R., and Strick, P. L. (1986). Parallel organization of functionally segregated circuits linking basal ganglia and cortex. Annu. Rev. Neurosci. 9, 357-381.

Ashburner, J. (2007). A fast diffeomorphic image registration algorithm. Neuroimage 38, 95-113.

Batterham, R. L., ffytche, D.H., Rosenthal, J. M.,Zelaya, F. O., Barker, G. J., Withers, D. J., and Williams, S.C. (2007). PYY modulation of cortical and hypothalamic brain areas predicts feeding behaviour in humans. Nature 450, 106-109.

Beasley, J. M., Ange, B. A., Anderson, C. A., Miller Iii, E. R., Holbrook, J. T., and Appel, L. J. (2009). Characteristics associated with fasting appetite hormones (obestatin, ghrelin, and leptin). Obesity (Silver Spring) 17, 349-354.

Bechara, A., Damasio, A. R., Damasio, H, and Anderson, S. W. (1994). Insensitivity to future consequences

the distribution of genders across lean and obese groups was not explicitly balanced, which may influence the results (Pannacciulli et al., 2006, 2007).

Because our study was cross-sectional, we are not able to make inferences about whether our findings reflect the cause or effect of obesity. It is evenly likely that brain structure predicts the development of obesity or that obesity, accompanied by altered eating behavior, causes brain structure to change. In the future, longitudinal studies may answer this open question.

In summary, we suggest that in both genders, differences of both the hedonic and homeostatic control systems may reflect a bias in eating behavior. Only in women, we show that obesity modulates the behavioral preference for salient immediate rewards in the face of negative long-term consequences. Since behavioral experiments and structural MRI were carried out on different samples (see Materials and Methods) we could not directly relate these behavioral differences to the structural alterations. However, we hypothesize that the additional structural differences seen in obese women can be interpreted as a reflection of behavior paralleling obesity, namely that behavioral control is progressively dominated by habit-like behavior as opposed to goal-directed actions. Furthermore, our findings may be important for the recognition of obesity as a form of addiction. Additional studies of gender differences in behavioral control will be important for investigating the etiology of eating and body-weight disorders and for designing gender-appropriate treatments (Raji et al., 2010).

\section{ACKNOWLEDGMENTS}

This work was supported by the Federal Ministry of Education and Research [BMBF: Neurocircuits in obesity to Annette Horstmann, Michael Stumvoll, Arno Villringer, Burkhard Pleger; IFB AdiposityDiseases (FKZ: 01EO1001) to Annette Horstmann, Jane Neumann, David Mathar, Arno Villringer, Michael Stumvoll] and the European Union (GIPIO to Michael Stumvoll). We thank Rosie Wallis for proofreading the manuscript.

responses to food. Physiol. Behav. 99, 538-543.

Daw, N. D., Niv, Y., and Dayan, P. (2005). Uncertainty-based competition between prefrontal and dorsolateral striatal systems for behavioral control. Nat. Neurosci. 8, 1704-1711.

Dileone, R. J. (2009). The influence of leptin on the dopamine system and implications for ingestive behavior. Int. J. Obes. 33, S25-S29.

Draganski, B., Gaser, C., Busch, V. Schuierer, G., Bogdahn, U., and May, A. (2004). Changes in grey matter induced by training newly honed juggling skills show up as a transient feature on a brain-imaging scan. Nature 427, 311-312.

Draganski, B., Kherif, F., Klöppel, S., Cook, P. A., Alexander, D. C., Parker, G. J., Deichmann, R., Ashburner, J., and Frackowiak, R. S. (2008). Evidence for segregated and integrative connectivity patterns in the human basal ganglia. J. Neurosci. 28, 7143-7152.

Edelsbrunner, M. E., Herzog, H., and Holzer, P. (2009). Evidence from knockout mice that peptide YY and neuropeptide $Y$ enforce murine locomotion, exploration and ingestive behaviour in a circadian cycle- and gender-dependent manner. Behav. Brain Res. 203, 97-107.

Farooqi, I. S., Bullmore, E., Keogh, J., Gillard, J., O'Rahilly, S., and Fletcher, P. C. (2007). Leptin regulates striatal regions and human eating behavior. Science 317, 1355.

Frank, M. J. (2009). Slave to the striatal habit (commentary on Tricomi et al.). Eur. J. Neurosci. 29, 2223-2224.

Frank, M. J., and Claus, E. D. (2006). Anatomy of a decision: striato-orbitofrontal interactions in reinforcement learning, decision making, and reversal. Psychol. Rev. $113,300-326$ 
Fulton, S., Pissios, P., Manchon, R. P., Stiles, L.,Frank, L., Pothos,E.N.,Maratos-Flier, E., and Flier, J. S. (2006). Leptin regulation of the mesoaccumbens dopamine pathway. Neuron $51,811-822$.

Greene, G. W., Schembre, S. M., White, A. A., Hoerr, S. L., Lohse, B., Shoff, S., Horacek, T., Riebe, D., Patterson, J., Phillips, B. W., Kattelmann, K. K., and Blissmer, B. (2011). Identifying clusters of college students at elevated health risk based on eating and exercise behaviors and psychosocial determinants of body weight. J. Am. Diet. Assoc. 111, 394-400.

Hayasaka, S., Phan, K. L., Liberzon, I., Worsley, K. J., and Nichols, T.E. (2004). Nonstationary cluster-size inference with random field and permutation methods. Neuroimage 22, 676-687.

Hommel, J. D., Trinko, R., Sears, R. M., Georgescu, D., Liu, Z. W., Gao, X. B., Thurmon, J. J., Marinelli, M., and DiLeone, R. J. (2006). Leptin receptor signaling in midbrain dopamine neurons regulates feeding. Neuron 51 , 801-810.

Horstmann, A., Frisch, S., Jentzsch, R. T., Müller, K., Villringer, A., and Schroeter, M. L. (2010). Resuscitating the heart but losing the brain: brain atrophy in the aftermath of cardiac arrest. Neurology 74, 306-312.

Jimura, K., Locke, H. S., and Braver, T. S. (2010). Prefrontal cortex mediation of cognitive enhancement in rewarding motivational contexts. Proc. Natl. Acad. Sci. U.S.A. 107, 8871-8876.

Koob, G. F., and Volkow, N. D. (2010). Neurocircuitry of addiction. Neuropsychopharmacology 35, 217-238.

Malik, S., McGlone, F., Bedrossian, D., and Dagher, A. (2008). Ghrelin modulates brain activity in areas that control appetitive behavior. Cell Metab. 7 , 400-409.

Marshall, J.A., Grunwald, G. K., Donahoo, W. T., Scarbro, S., and Shetterly, S. M. (2000). Percent body fat and lean mass explain the gender difference in leptin: analysis and interpretation of leptin in Hispanic and non-Hispanic white adults. Obes. Res. 8, 543-552.

Miller, E. K., and Cohen, J. D. (2001). An integrative theory of prefrontal cortex function. Annu. Rev. Neurosci. 24, 167-202.

Mueller, K., Anwander, A., Möller, H. E., Horstmann, A., Lepsien, J., Busse, F., Mohammadi, S., Schroeter, M. L., Stumvoll, M., Villringer, A., and Pleger, B. (2011). Sex-dependent influences of obesity on cerebral white matter investigated by diffusion-tensor imaging. PLoS ONE 6, e18544. doi: 10.1371/ journal.pone. 0018544

Nederkoorn, C., Smulders, F. T., Havermans, R. C., Roefs, A., and Jansen, A. (2006). Impulsivity in obese women. Appetite 47, 253-256.

Pannacciulli, N., Del Parigi, A., Chen, K., Le, D. S., Reiman, E. M., and Tataranni, P. A. (2006). Brain abnormalities in human obesity: a voxel-based morphometric study. Neuroimage 31, 1419-1425.

Pannacciulli, N., Le, D. S., Chen, K. Reiman, E. M., and Krakoff, J. (2007). Relationships between plasma leptin concentrations and human brain structure: a voxel-based morphometric study. Neurosci. Lett. 412, 248-253.

Parigi, A. D., Chen, K., Gautier, J. F., Salbe, A. D., Pratley, R. E., Ravussin, E., Reiman, E. M., and Tataranni, P. A. (2002). Sex differences in the human brain's response to hunger and satiation. Am. J. Clin. Nutr. 75 1017-1022.

Park, K. G., Park, K. S., Kim, M. J., Kim, H. S., Suh, Y. S., Ahn, J. D., Park, K. K., Chang, Y. C., and Lee, I. K. (2004). Relationship between serum adiponectin and leptin concentrations and body fat distribution. Diabetes Res. Clin. Pract. 63, 135-142.

Philpot, K. B., Dallvechia-Adams, S., Smith, Y., and Kuhar, M. J. (2005). A cocaine-and-amphetamine-regulated-transcript peptide projection from the lateral hypothalamus to the ventral tegmental area. Neuroscience $135,915-925$

Plassmann, H., O’Doherty, J. P., and Rangel, A. (2010). Appetitive and aversive goal values are encoded in the medial orbitofrontal cortex at the time of decision making. J. Neurosci. 30, 10799-10808.

Provencher, V., Drapeau, V., Tremblay, A., Després, J. P., and Lemieux, S. (2003). Eating behaviors and indexes of body composition in men and women from the Québec family study. Obes. Res. 11, 783-792.

Raji, C. A., Ho, A. J., Parikshak, N. N., Becker, J. T., Lopez, O. L., Kuller, L. H., Hua, X., Leow, A. D., Toga, A. W., and Thompson, P. M. (2010). Brain structure and obesity. Hum. Brain Mapp. 31, 353-364.

Rolls, B. J., Fedoroff, I. C., and Guthrie, J. F. (1991). Gender differences in eating behavior and body weight regulation. Health Psychol. 10, 133-142.

Rothemund, Y., Preuschhof, C., Bohner G., Bauknecht, H. C., Klingebiel,
R., Flor, H., and Klapp, B. F. (2007) Differential activation of the dorsal striatum by high-calorie visual food stimuli in obese individuals. Neuroimage 37, 410-421.

Schäfer, A., Vaitl, D., and Schienle, A. (2010). Regional grey matter volume abnormalities in bulimia nervosa and binge-eating disorder. Neuroimage 50 , 639-643.

Scholz, J., Klein, M. C., Behrens, T. E., and Johansen-Berg, H. (2009). Training induces changes in whitematter architecture. Nat. Neurosci. 12, 1370-1371.

Schwartz, M. W., Peskind, E., Raskind, M., Boyko, E. J., and Porte, D. (1996). Cerebrospinal fluid leptin levels: relationship to plasma levels and to adiposity in humans. Nat. Med. 2 589-593.

Sluming, V., Barrick, T., Howard, M., Cezayirli, E., Mayes, A., and Roberts, N. (2002). Voxel-based morphometry reveals increased gray matter density in Broca's area in male symphony orchestra musicians. Neuroimage 17 1613-1622.

Smeets, P. A., de Graaf, C., Stafleu, A., van Osch, M. J., Nievelstein, R. A., and van der Grond, J. (2006). Effect of satiety on brain activation during chocolate tasting in men and women. Am. J. Clin. Nutr. 83, 1297-1305.

Stanek, K. M., Grieve, S. M., Brickman, A. M., Korgaonkar, M. S., Paul, R. H., Cohen, R. A., and Gunstad, J. J. (2011) Obesity is associated with reduced white matter integrity in otherwise healthy adults. Obesity (Silver Spring) 19, 500-504.

Taki, Y., Kinomura, S., Sato, K., Inoue, K., Goto, R., Okada, K., Uchida, S., Kawashima, R., and Fukuda, H. (2008). Relationship between body mass index and gray matter volume in 1,428 healthy individuals. Obesity (Silver Spring) 16, 119-124.

Taubert, M., Draganski, B., Anwander, A., Müller, K., Horstmann, A. Villringer, A., and Ragert, P. (2010). Dynamic properties of human brain structure: learning-related changes in cortical areas and associated fiber connections. J. Neurosci. 30 11670-11677.

Tricomi,E., Balleine, B.W., and O'Doherty, J. P. (2009). A specific role for posterior dorsolateral striatum in human habit learning. Eur. J. Neurosci. 29, 2225-2232.

Uher, R., Treasure, J., Heining, M., Brammer, M. J., and Campbell, I. C. (2006). Cerebral processing of food- related stimuli: effects of fasting and gender. Behav. Brain Res. 169, 111-119.

Volkow, N. D., and Wise, R. A. (2005). How can drug addiction help us understand obesity? Nat. Neurosci. 8, 555-560.

Walther, K., Birdsill, A. C., Glisky, E. L., and Ryan, L. (2010). Structural brain differences and cognitive functioning related to body mass index in older females. Hum. Brain Mapp. 31, 1052-1064.

Wang, G. J., Volkow, N. D., Telang, F., Jayne, M., Ma, Y., Pradhan, K., Zhu, W. Wong, C. T., Thanos, P. K., Geliebter, A., Biegon, A., and Fowler, J.S. (2009). Evidence of gender differences in the ability to inhibit brain activation elicited by food stimulation. Proc. Natl. Acad. Sci. U.S.A. 106, 1249-1254.

Wang, J., Korczykowski, M., Rao, H., Fan, Y., Pluta, J., Gur, R. C., McEwen, B. S. and Detre, J. A. (2007). Gender difference in neural response to psychological stress. Soc. Cogn. Affect. Neurosci. 2, 227-239.

Weller, R. E., Cook, E. W., Avsar, K. B., and Cox, J. E. (2008). Obese women show greater delay discounting than healthy-weight women. Appetite 51, 563-569.

World Health Organization. (2010). WHO Global Infobase. Geneva: World Health Organization.

Yin, H. H., and Knowlton, B. J. (2006). The role of the basal ganglia in habit formation. Nat. Rev. Neurosci. 7, 464-476.

Conflict of Interest Statement: The authors declare that the research was conducted in the absence of any commercial or financial relationships that could be construed as a potential conflict of interest.

Received: 06 April 2011; accepted: 26 May 2011; published online: 10 June 2011. Citation: Horstmann A, Busse FP, Mathar D, Müller K, Lepsien J, Schlögl H, Kabisch $S$, Kratzsch J, Neumann J, Stumvoll M, Villringer A and Pleger B (2011) Obesityrelated differences between women and men in brain structure and goal-directed behavior. Front. Hum. Neurosci. 5:58. doi: 10.3389/fnhum.2011.00058

Copyright (c) 2011 Horstmann, Busse, Mathar, Müller, Lepsien, Schlögl, Kabisch, Kratzsch, Neumann, Stumvoll, Villringer and Pleger. This is an open-access article subject to a non-exclusive license between the authors and Frontiers Media SA, which permits use, distribution and reproduction in other forums, provided the original authors and source are credited and other Frontiers conditions are complied with. 